\title{
PREFLARE ACTIVITY
}

V. GAIZAUSKAS

Herzberg Institute of Astrophysics, National Research Council of Canada, Ottawa, Canada K1A OR6

\begin{abstract}
Magnetic reconnection at current sheets or in current-bearing arches in the solar atmosphere is generally accepted as the mechanism responsible for the sudden energy release in solar flares. Attempts have so far been unsuccessful to isolate from the observations some unique preconditions which would be necessary and sufficient to ensure rapid conversion of energy by this process. Here we survey recent multi-wavelength observations which illustrate the variety of preflare activity. Multiple structures are now believed to participate in the energy release. Dynamic global coupling of the magnetic fields between a flaring site and the rest of an activity complex is seen from the data to be an important aspect of preflare activity.
\end{abstract}

\section{Introduction}

It is generally accepted that flares draw their power from the free energy stored in stressed magnetic fields (Švestka, 1976). The rapid transformation of magnetic energy into plasma heating and particle acceleration is believed to occur through magnetic reconnection when the stresses exceed a critical threshold. A possible alternative is the formation of double layers (see Kuijpers, these proceedings). Reconnection mechanisms may involve magnetic tearing of single coronal loops (Spicer, 1976; Van Hoven, 1976) or the merging of current sheets (see Priest, 1985, for an extended review). Other plausible mechanisms incorporated into flare models have been categorized according to their drivers by Spicer and Brown (1981). By studying the preflare state we hope to constrain the choice of driving mechanisms, always bearing in mind that different regimes of plasma physics may apply as the flare evolves from the preflare to the impulsive phase (Van Hoven and Hurford, 1986). The most important questions to be settled from preflare observations concern the geometry of the magnetic field, the stresses applied to them from photospheric to coronal heights, and the changing physical properties of the plasma trapped in those fields.

Because the transition is not perfectly abrupt, there is always some arbitrariness about the state, preflare or flaring, to which a particular phenomenon belongs. The difficulty is aggravated by the fact that phenomena assigned to the preflare phase do occur even in the absence of any flare. The problem is illustrated by the idealized flux curve in Figure 1 which is adapted from actual cm-wavelength observations of a solar flare. Without spatial resolution, e.g., observing a stellar flare, there is no certainty that the small transient labelled 'precursor' has anything to do with the flare itself. This type of discrete transient will be discussed in Section 2 along with phenomena, sometimes offered as evidence for preheating, which might produce a small plateau as the emission begins to rise (Figure 1).

In Figure 1 the weak sporadic emission labelled 'preflash', part of the onset of the flare, signals the initial release of high-energy particles. It is manifested as decimetric radio pulsations, metric type III bursts, and hard as well as soft X-rays (Benz et al.,

(C) 1989 Kluwer Academic Publishers. Printed in Belgium. 


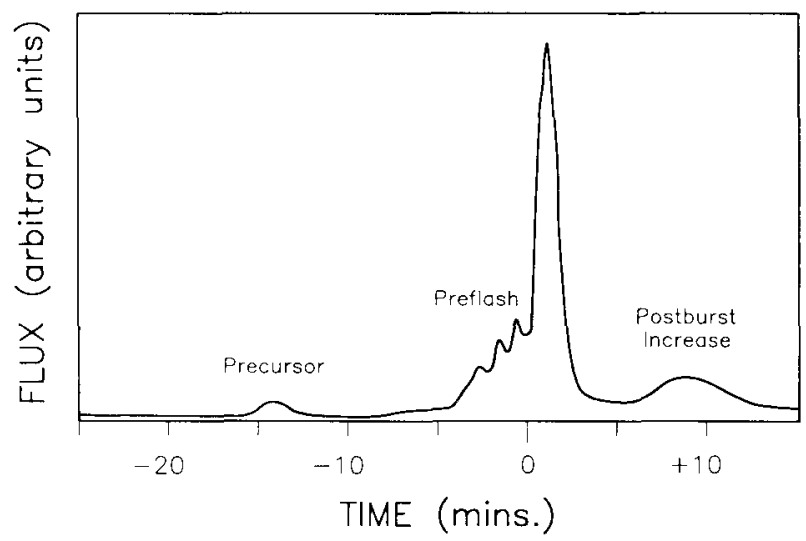

Fig. 1. Idealized flux profile of a flare detected in an energy band which senses a distinct impulsive phase at $t=0$.

1983; Raoult et al., 1985; Machado, Orwig, and Antonucci, 1986; Kundu et al., 1987). These spatially-resolved observations suggest that a morphological transition, involving different plasmas, occurs between the onset and impulsive phases of a flare. A comprehensive study of X-ray emission in flares by Machado et al. (1988) is explicit on this point. The basic structure of a flare consists of an initiating bipole impacted against one or more adjacent bipoles. During flare onset the X-ray emission is concentrated in the initiating bipole, but during the impulsive phase most of the energy is released inside the initiating bipole and/or inside one of the adjacent bipoles rather than at the point of impact between them. The timing and nature of these phenomena implies a continuous process of acceleration that starts before the flash phase and continues beyond the impulsive phase of the flare (Vlahos et al., 1986).

Even though they precede the impulsive phase by as much as a few minutes, the intimate relation of preflash phenomena to the main energy release of flare energy sets them outside the scope of this paper. Emphasis will be given instead in Section 3 to evolutionary processes on the scale of hours and even days which are linked to the storage of excess energy at the site of a flare. Because more detailed evidence has been recently acquired on the most distinctive type of preflare event, the erupting filament, all of Section 4 is devoted to a discussion of the changes preceding three eruptions.

Although this review deals exclusively with examples of solar flares, global interactions are stressed wherever possible as having possible application to other stars.

\section{Precursors and Preheating}

A precursor is here defined as a transient event preceding the impulsive phase, possibly even before the onset and not necessarily at the site of the flare itself (Priest et al., 1986, Section 1.4). Under this broad definition, earlier flares can qualify as precursors because their frequency of occurrence may sometimes reach a crescendo just before a major flare (Dodson and Hedeman, 1976). We include two special cases: earlier flares in the same 
location and with similar emission patterns (homologous flares); earlier flares in different locations but erupting in near synchronism (sympathetic flares).

\subsection{Homologous flares}

Homology is commonly found during periods of frequent flare activity (Woodgate, 1982; Zirin, 1983). We can infer that the same flare-producing stresses act on magnetic field configurations for hours, sometimes days. The rate of repetition of flares belonging to the same homologous series can be as rapid as a few per hour or as infrequent as one in several days (Martres et al., 1984). But no consistent relationship has been found during SMY between the repetition rate of homologous flares and the brightness of their X-ray, optical, or microwave emissions (Gaizauskas, 1982; Woodgate, 1982). The stresses within an active region need not, therefore, be uniformly applied with time. Indeed, Machado (1985) finds that homology is clearly correlated with the development of magnetic shear in an active region.

The comparison of several series of recurrent events should isolate essential preflare factors, but that hope is only partially realized. Woodgate et al. (1984) find that flow patterns and magnetic shear qualify for an essential role in some but not every homologous set which they investigated. Strict homology, in terms of identical size, shape and location of flare kernels, is never realized because of the incessant evolution of magnetic fields on a small scale (Gaizauskas, 1982; Zirin, 1983; Machado, 1985).

\subsection{SYMPATHETIC FLARES}

Flare-related linkages between adjacent and even remote active regions ought to be a natural consequence of the interwoven loop structure of the corona as validated by Skylab. A single excitation could in principle be transmitted along a multiplicity of linkages and produce flare-like responses at several remote sites. Different statistical tests of large samples of flares have however led to opposite conclusions: no significant increase in the excess beyond the random rate of flares coinciding to within 20 min for pairs of active regions more than $30^{\circ}$ apart (Fritzová-Švestková, Chase, and Švestka, 1976); a significant correlation between the maximum and onset phases of flares in solar regions spaced from $19^{\circ}$ up to $104^{\circ}$ apart (Ogir, 1981).

Case studies of flares using modern mapping methods in microwaves and in X-rays now show energy being transferred between sites in adjacent active regions. Kundu, Rust, and Bobrowsky (1983) estimate a velocity $\geq 6000 \mathrm{~km} \mathrm{~s}^{-1}$ for $6-\mathrm{cm}$ emission advancing along a loop $\sim 10^{5} \mathrm{~km}$ long joining faint flare-like $\mathrm{H} \alpha$ brightenings. The simultaneous microwave and X-ray observations of Kundu et al. (1984) find an X-ray brightening advancing along a loop $1.3 \times 10^{5} \mathrm{~km}$ long joining two regions in the same complex of activity. The speed of the X-ray front $\left(\sim 100 \mathrm{~km} \mathrm{~s}^{-1}\right)$ and its orientation are consistent with a disturbance propagating between the two subflares which occurred with a delay of $\sim 25 \mathrm{~min}$ near opposite footpoints of the interconnecting loop. Double microwave events at $1.8 \mathrm{~cm}$ wavelength, selected for the similarity of the time profiles of both components, have been studied by Nakajima et al. (1985). Their spatiallyresolved data show that a secondary microwave burst can occur at a distance of 
$10^{5}-10^{6} \mathrm{~km}$ from the primary flare site. For two of these events a new X-ray source was observed adjacent to the secondary source and was associated with faint $\mathrm{H} \alpha$ brightenings. The velocity deduced from the measurements $\left(\gg 10^{4} \mathrm{~km} \mathrm{~s}^{-1}\right)$ implies that the exciting agent must be high-energy electrons produced at the primary flare site. This is unequivocal evidence that one flare can trigger another, albeit a weak one, at a remote site.

\subsection{SOFT X-RAY PRECURSORS}

Transient enhancements in soft X-rays commonly appear in data from Skylab (Kahler, 1979; Webb, 1985) and from SMM (Section 1.4 of Priest et al., 1986) as loops or kernels close to, but not necessarily at, flare sites for many minutes preceding the impulsive phase. A soft X-ray precursor may coincide in place and shape with features belonging to the impulsive phase as in the case of a limb flare observed by de Jager et al. (1983). Spatial coincidence between x-ray precursor and flare is usually partial, a situation which is consistent with flare energy being released within two or more interacting magnetic loops (Machado et al., 1988).

Flare-related coronal mass ejections (CMEs) support the view that the interaction between magnetic loops is a basic ingredient of the flare process. Weak soft X-ray bursts have been observed at a time coincident with the projected onset of a CME associated with a flare; precursor bursts occur some tens of minutes prior to the impulsive phase and are located in one foot of a pre-existing large coronal arch (Harrison et al., 1985; Simnett and Harrison, 1985; Harrison, 1986). The rest of the arch brightens weakly within minutes. Outward motion of plasma emitting X-rays during the precursor burst indicates that a CME has been launched (Harrison et al., 1985; Harrison, 1986). Because the associated flares sit to one side of the centreline of the ejecta, Harrison proposes that (i) a small magnetic feature interacts with one foot of a large pre-existing arch; (ii) a CME is launched without an explosive release of energy; (iii) a flare is subsequently triggered at the site of the precursor by prevailing post-launch conditions. It is worth noting that neither radio emission nor hard X-rays $\geq 15 \mathrm{keV}$ have been associated with these soft X-ray precursors (Simnett and Harrison, 1985).

\subsection{MicrowaVe PRECURSORS}

Radio precursors were discovered long ago to consist of changes in intensity and/or polarization of the microwaves emitted from an active region some tens of minutes before the onset of a flare (see Kundu, 1965, and references therein). Recent samplings of many flares indicate, however, that radio precursors do not occur in a majority of cases. For bursts at $17 \mathrm{GHz}$ with a peak flux density $>10$ s.f.u., the probability of weaker preceding activity is only $25 \%$ (Kai, Nakajima, and Kosugi, 1983). The probability increases to $\sim 50 \%$ if much stronger bursts ( $>500$ s.f.u. at $17 \mathrm{GHz}$ ) are selected. The average delay between precursor and main bursts is $25 \mathrm{~min}$. In samples of major flares observed interferometrically at $10.7 \mathrm{GHz}$, Hurford and Zirin (1982) find distinctive changes to occur in only $11 \%$ of their cases a few minutes to tens of minutes before the beginning of the impulsive phase. The most common signature is a step-like increase 
in amplitude accompanied by a decrease or a reversal in the degree of polarization. Out of eight bursts observed with supersynthesis arrays at 2,6 , and $20 \mathrm{~cm}$ wavelength by Willson (1983) only one exhibits detectable preburst heating. Taken together, these results with different instruments suggest that precursors are not a general feature of the burst process at centimeter wavelengths. But this conclusion should be treated with caution because, as Hurford, Read, and Zirin (1984) show, pre-impulsive bursts may occur in such narrow spectral bands that they can easily be missed by instruments responding to a single frequency.

Those radio precursors which are observed at high spatial resolution provide important insights to the initiation of a flare. Morphological transitions of the emitting structures, continuing for minutes to tens of minutes through the preflare and impulsive phases, are a sign of interacting, pre-existing loops (Kundu et al., 1982; Willson and Lang, 1984; Kundu and Shevgaonkar, 1985; Lang and Willson, 1986). Dramatic changes in polarization are observed at centimeter wavelengths to begin from minutes up to an hour before a flare and to continue through the impulsive phase (Lang, 1979; Kundu et al., 1982; Kundu, Schmahl, and Velusamy, 1982; Willson and Lang, 1984; Kundu, 1986). The preflare reorganization of magnetic field implied by these results can be interpreted in different ways: as propagation effects in a highly structured and highly dynamic magnetoionic plasma (Willson, 1983; Van Hoven and Hurford, 1986); as the appearance of new magnetic structures low in the corona; or in some cases simply as sources switching on and off at different times and at different locations within a magnetically complex region. It is tempting to explain the polarization changes in terms of new magnetic flux emerging from beneath the solar surface (Kundu, 1986), but concomitant observations of the lower atmosphere on a fine scale are rare. Indeed in the one case where observations of changing polarization at centimeter wavelengths are reinforced by optical observations at high spatial and temporal resolution, none of the usual signatures of emerging flux are detected (see below, Section 4.3).

\subsection{UltRAVIOLET PRECURSORS}

Transient brightenings of UV lines over a broad range of amplitudes are common on a small spatial scale above active regions (Van Hoven et al., 1980; Cheng et al., 1981; Cheng, Tandberg-Hanssen, and Orwig, 1984; Porter, Toomre, and Gebbie, 1984; Cheng and Tandberg-Hanssen, 1986; Priest et al., 1986, Section 1.4.4). Some of the UV kernels with intense preflare activity become flares. But others do not join in a later flare which may be concentrated nearby in entirely new kernels (Cheng et al., 1981; Cheng, Tandberg-Hanssen, and Orwig, 1984). A change in the frequency of small-scale events before and after a flare has been sought with inconclusive results owing to inadequate statistics (Porter, Toomre, and Gebbie, 1984).

In those few cases of good spatial and temporal coverage obtained simultaneously at multiple wavelengths, transient UV preflare activity occurs in very inhomogeneous surroundings. The limb flare of 30 April, 1980 erupted at the junction of a small loop rising into an overlying structure (Woodgate et al., 1981; de Jager et al., 1983). Strong $\mathrm{UV}$ and X-ray brightenings at the footpoint of the rising loop precede that flare. 
Repeated transient UV brightenings are associated with the upwelling, twisting, and disruption of an active-region filament in the 20 min leading up to a 2-ribbon flare (Kundu et al., 1985). Differences between preflare UV structures from one flare to the next impede our ability to designate a reliable precursor against the varying UV background.

\subsection{Preflare heating}

Current sheet models (e.g., Heyvaerts, Priest, and Rust, 1977) and the unstable arch model (Spicer, 1976) of flares predict a pre-heating phase. Analysis of spatiallyunresolved soft $\mathrm{X}$-ray emission from many flares observed by an early satellite indicates a tendency for $\mathrm{X}$-ray bursts to begin, on the average, about two minutes earlier than their associated $\mathrm{H} \alpha$ flares (Thomas and Teske, 1971). Recent case studies of spatiallyresolved events do show pre-heated structures many minutes before they flare at microwave frequencies (Kundu et al., 1982; Lang and Willson, 1984; Willson, 1984; Kundu and Shevgaonkar, 1985) and in the EUV (Cheng et al., 1982, 1983).

Surveys of many Skylab soft X-ray images do not, however, indicate a requirement for coronal preflare heating lasting longer than $2 \mathrm{~min}$ in small flares consisting of just one or two loops (Kahler, 1979); they do reveal cases where structures displaced from the flare site brighten tens of minutes before onset (Webb, 1985). In a complementary study of a randomly selected set of moderate-sized flares observed with $\sim 2.5$ arc min resolution by the Mapping X-ray Heliometer on OSO-8, Wolfson (1982) finds intensity variations in soft $\mathrm{X}$-rays before a flare to be no different from the variations in the same active region when no flare occurs. Furthermore, slowly varying emission in harder $\mathrm{X}$-rays $(3.5-8 \mathrm{keV})$ occurs even in the absence of flares and is located predominantly over polarity inversion lines where volumes of hot plasma have temperatures exceeding $10^{7} \mathrm{~K}$ (Schadee, de Jager, and Švestka, 1983). These facts support Kahler's (1979) position that high temperatures and /or densities in the preflare region are not generally pre-requisites for a flare.

\subsection{SURGING ARCHES}

In a sample of 58 flares, Mouradian, Martres, and Soru-Escaut (1983) find that just over half are preceded by a surging arch - a transient absorbing feature visible in solar regions at wavelengths displaced from the central core of $\mathbf{H} \alpha$. Red- and blue-shifted components are visible simultaneously from the first appearance of the structure; they are not cospatial. Initially linear in shape, the structure arches expands rapidly and becomes a complex assortment of multiple strands about the time the associated flare erupts in the same active region. The average delay between the beginning of the surging arch and the flare is $11 \mathrm{~min}$ in this sample. An early example of this phenomenon was noted by Athay and Moreton (1961) in conjuction with a blast wave produced by a flare (the same event is illustrated in more detail by Moreton, 1961). The relationship of the surging arch, presumably a rising magnetic loop, to the short-term evolution of the local magnetic field and, hence, to the flare itself is still obscure.

The rich variety of precursors surveyed above underscores the complexity of the preflare state. A typical signature of that state cannot yet be isolated from existing observations of precursors. 


\section{Evolution of Magnetic Fields}

Flare activity is intimately connected with evolving magnetic fields (see reviews and references therein by Martin (1980) and by Priest et al. (1986)). Changing fields can create a current sheet (e.g., Heyvaerts, Priest, and Rust, 1977) and current networks (e.g., Hénoux and Somov, 1987). The destabilization of these current-forming processes can be initiated by a sudden injection of a new magnetic bipole. But it can also result from steady evolutionary trends which often get obscured by numerous short-lived changes in magnetic structures. We examine below the emergence of magnetic flux and the creation of magnetic shear, two factors commonly associated with flares, within the context of magnetic evolution on the Sun.

\subsection{EMERGING FLUX AND MAGNETIC COMPLEXITY}

Numerous flares erupt early in the development of an Emerging Flux Region (EFR), but they are minor in the case of a new region growing in isolation (Bruzek, 1967). The large flares with the interesting physics erupt where old and new magnetic flux interact. Interactions are frequent because active regions do not form at random on the Sun. They tend to appear in clusters called 'complexes' or 'nests' of activity which survive for at least several rotations (Gaizauskas et al., 1983; Castenmiller, Zwaan, and van der Zalm, 1986). During its active lifetime, an activity complex will be refreshed by injections of new magnetic flux in the form of bipolar active regions at a rate equivalent to their disappearance; the mean lifetime of a single bipole is about 2 weeks. Thus the detection on a remote star of a large magnetic structure need not be interpreted as one immense starspot; it may be instead an agglomeration of many spots.

Complexes are illustrated in the magnetograms of Figure 2 at the same solar longitude in the southern hemisphere on successive rotations of the Sun in 1980. The elongation of the activity complex in the June 22 magnetogram (right) is created by the emergence and expansion of 17 small- to medium-sized bipolar regions along the inclined polarity inversion line where previously there had been just three large regions. During the May rotation (left) only one new region forms in the complex during its entire disk passage. The day following its appearance is marked by intense flare activity centered on the small new region. During the June rotation two-thirds of the flaring sites are located in or on the boundaries of the 17 new regions (Martin et al., 1982). Yet the level of flare activity measured as counts of events or as total peak emission in soft X-rays is comparable for both rotations (Gaizauskas, 1982). If emerging flux in itself always dominates the flare process, the level of flaring during the second rotation should greatly exceed that of the first. Since this does not happen, the individual flux emergences must fulfill other conditions in order to be flare-effective.

The single small region that emerged on the first rotation contributed a disproportionate share of the strong flares from the activity complex. Within the single penumbra comprising that small region, pores of opposite magnetic polarity were arranged in a complex pattern and were moving rapidly $\left(>0.1 \mathrm{~km} \mathrm{~s}^{-1}\right)$ relative to each other (Nagy, 1983). A similar situation arose on a much greater scale in McMath 15403, 


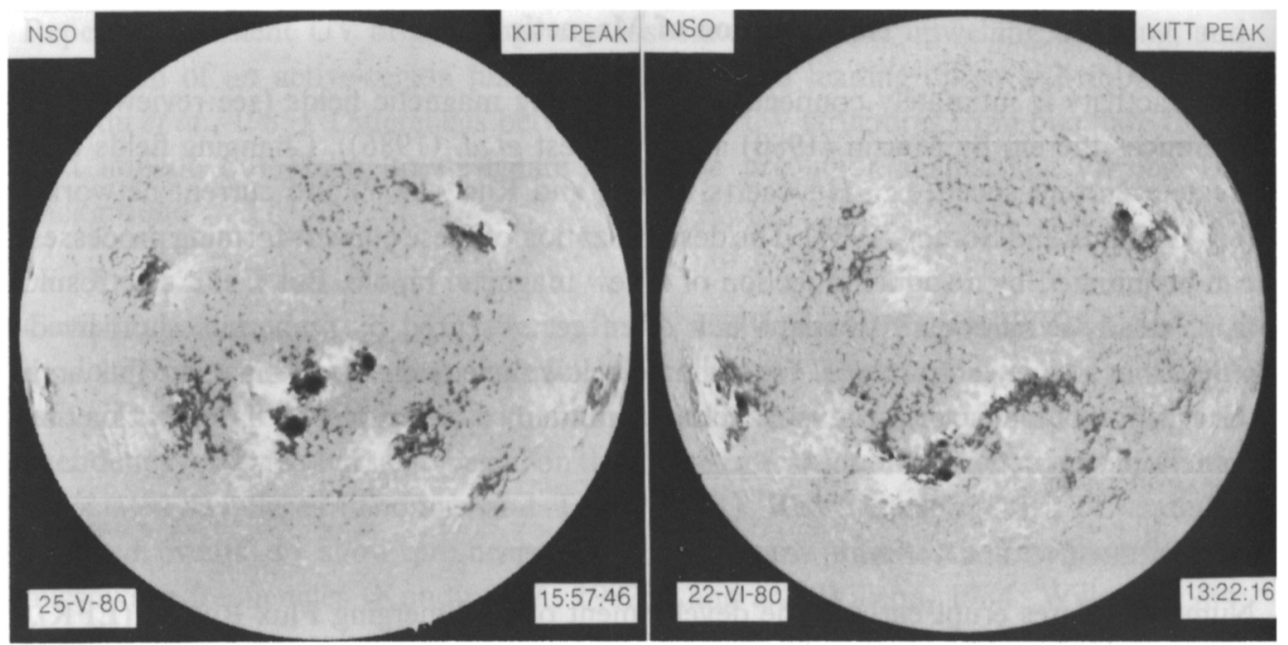

Fig. 2. A large complex of activity (sunspot 'nest') crossing the central meridian on successive solar rotations in May and June 1980. Orientation: north is at the top, and west is to the right. Whole-disk magnetograms from the National Solar Observatory supplied courtesy of J. W. Harvey.

a region which by itself produced $10 \%$ of the 324 flares emitting soft X-rays stronger than class M1 in a two-year period studied by Gaizauskas and McIntosh (1986b). A large new sunspot group formed inside an existing large spot on $10 \mathrm{July,} 1978$ (Sattarov, 1983). The expansion of the complex magnetic pattern resulting from this superposition in McMath 15403 (Dezsö et al., 1980) was accompanied by one of the strongest flaring episodes of Cycle 21.

Extreme magnetic complexity which has long been recognized as advantageous for producing great flares (Giovanelli, 1939) is a consequence of the Sun's tendency to keep pushing magnetic flux up within long-lived nests of activity. Observations show that the complexity can be prefabricated before any spots emerge (Zirin and Tanaka, 1973), or it can be created after originally unpaired bipoles emerge and push together as they grow (Tang, 1983; Zirin and Liggett, 1987). Flare activity stays enhanced in a nest of activity during its initial one or two disk passages but diminishes thereafter (Gaizauskas and McIntosh, 1986b).

Bai (1988) has examined recurrent flare activity using data from three solar cycles. $\mathrm{He}$ finds that superactive regions with major flares appear more frequently in certain areas on the Sun which he calls 'hot spots'. The hot spots are active intermittently and can be traced from one sunspot cycle to the next. Bai attributes hot spots to long-lived subsurface activity which rotates rigidly but at slightly different rates in the northern and southern hemispheres.

Proof of existence of a long-lived global pattern of active sources would eventually simplify flare prediction. Clues to a large-scale pattern can be found in the discoveries by Soru-Escaut, Martres, and Mouradian (1985) and by Mouradian et al. (1987) of a relation between long-lived $\mathrm{H} \alpha$ filaments with anomalous rates of rotation, the emergence of active centers, and the flare productivity in those centers. McIntosh and Wilson 
(1985) present additional observational evidence which suggests that large-scale solar magnetic fields are organized as cellular patterns with sunspot regions forming on long-lived, updrafting boundaries of the cells. They claim that the trajectories of $\mathrm{H} \alpha$ filaments which last for many solar rotations can be used to define areas of enhanced shear and vorticity in the local flow which are later identified with the location of large flare-active regions.

In summary, the appearance and growth of new magnetic flux is a necessary precondition for flares. Small flares are common during the early phase of an EFR; large flares are often rooted in new, rapidly growing and complex patterns of magnetic flux. But the vast bulk of magnetic flux appears at the surface without producing flares as strong as an $\mathrm{Ml}$ event in X-rays - only $8 \%$ of the active regions met this condition in the two-year period studied by Gaizauskas and McIntosh (1986b). The proximity, orientation, and motion of the new flux relative to existing flux in an activity complex have, therefore, to meet stringent conditions in order to stimulate powerful flares.

\subsection{MAGNETIC SHEAR AND ELECTRIC CURRENTS}

The growth of an EFR inside an activity complex comprising several bipolar regions deforms the composite magnetic field of the system. The work done during the reconfiguration of the magnetic field into a non-potential form becomes available as free energy to power flares wherever and whenever a fast and efficient dissipation mechanism can be activated. Processes for transforming a quasi-stable magnetic configuration from a passive to a highly dynamic state are reviewed in Priest (1981) and by Spicer (1982). Here we draw attention to some observations of the quasi-static preflare state which are relevant to setting initial conditions in models of these processes.

From the alignment of chromospheric fibrils adjacent to magnetic polarity inversions in active regions, it is commonly inferred that the magnetic field must be sheared along and above the inversion lines. Direct confirmation of magnetic shear at the photospheric level comes from measurements with vector magnetographs of the transverse component of the magnetic field. The azimuthal difference between the observed field and the calculated potential field is a measure of the degree of magnetic shear. Shear so-defined attains maximum values at the sites of flare onset located along the magnetic polarity inversion line in the active region studied by Hagyard et al. (1984). The frequency and magnitude of flares increases in accord with the growth and relaxation of the shear in this same active region (Krall et al., 1982). Rapid spot motions and high inferred velocity shear also coincide with increased flare activity (Krall et al., 1982; Gesztelyi and Kalman, 1986; Kovacs and Dezsö, 1986). Analysis of several active regions shows, however, that sites of strong magnetic shear can be created and persist along their magnetic inversion lines without becoming sites of flare kernels (Athay, Jones, and Zirin, 1986; Hagyard and Rabin, 1986). The concept of a critical shear as a preflare threshold seems not to be generally valid. The formation of flare-related shear has been observationally associated with flux emergence (Zirin, 1983), flux submergence (Rabin, Moore, and Hagyard, 1985), flux cancellation (Martin, Livi, and Wang, 1985), and vortical motions in EFR (Martres et al., 1982). But a flux emergence leading to the 
direct and long-enduring collision of two large sunspot umbrae of opposite polarity was not flare-effective until the interconnecting fibrils indicated a sudden transition from a current-free to a sheared magnetic configuration (Gaizauskas and Harvey, 1986a).

The peak electric current along the line-of-sight derived from vector magnetograms coincides exactly with the sites of flare initiation in a five-kernel flare observed by Lin and Gaizauskas (1987). Using photospheric and chromospheric data for the same event, Hagyard (1988) was able to model the current-bearing structures at the site of flare onset: two arcades of loops with a radius of only $45 \mathrm{~km}$, each carrying currents of $15 \times 10^{10} \mathrm{~A}$, and oriented about $60^{\circ}$ to the inversion line. Interacting loops on this scale cannot account for all of the observed kernels or their distribution. For this same active region, longer loops interconnecting other flare sites have been inferred from X-ray and chromospheric data (Machado et al., 1983; Ding et al., 1987).

Multi-kernel flares imply a global network of currents coupling different parts of activity complexes. The formation of multiple current sheets in the corona by systematic photospheric motions has been modelled for the case of a quadrupolar field (Baum and Bratenahl, 1980; Hénoux and Somov, 1987; Low and Wolfson, 1988). An example is shown in Figure 3 of the current system formed when two bipoles are coaligned

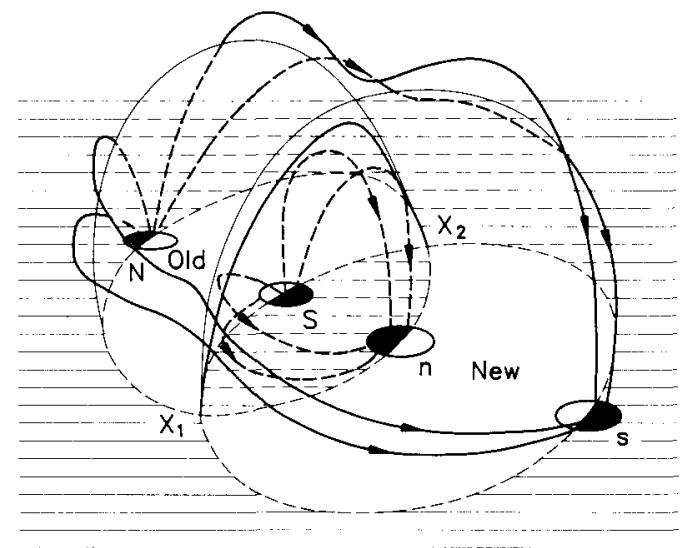

Fig. 3. Three-dimensional representation of coronal currents and magnetic field separatrix surfaces for a quadrupole. The magnetic bipoles depict a new active region forming in line with an old one. The heavy arc joining $X_{1}$ and $X_{2}$ is the separator between two cells of magnetic flux. Adapted from Hénoux and Somov (1987).

end-to-end. The coronal field is partitioned into flux cells by special surface distributions of magnetic field lines called separatrices. The ovoid-shaped separatrices intersect along separator lines, regions of interaction between current systems generated by footpoint motions in the separatrix cells. Reconnection at the separator may release energy not in a confined region but over entire separatrix surfaces, thereby affecting many magnetic field loops. The energy stored in both the separator current and the individual loops when released forms multi-kernel flares in the process. The significant point for flare build-up is that the current along the separator can be increased beyond an instability threshold by an excess relative to a potential field of magnetic flux in one of the separatrix 
cells and a deficiency in another, or by excessive kinetic energy (i.e., footpoint motions) in any cell. In this formulation of the problem, boundary conditions at remote parts of an activity complex cannot be ignored relative to the local conditions at the site of a flare.

\section{Erupting Filaments}

Enhanced mass motions in active-region filaments are one of the best preflare indicators. The association of erupting filaments with two-ribbon flares receives considerable attention because the geometry of the situation lends itself to theoretical modelling with predictive capabilities. It must be emphasized, however, that not all large flares are accompanied by erupting filaments, nor do all flare-related filament eruptions have a preflare phase. The reader is referred to Martin (1980) for a review of the observational aspects of filament activation and to Low (1982) and Priest (1982) for theoretical discussions of the stability of these structures.

A specific mechanism which is often invoked to destabilize a filament is reconnection at a current sheet between newly emerging or evolving flux and an overlying filament (Heyvaerts, Priest, and Rust, 1977). A quantitative verification of this model is difficult because changes in magnetic flux on a small scale are constantly happening against a backdrop of slowly-evolving magnetic patterns in any well-developed activity complex. Three specific examples of flare-related eruptions are discussed below to illustrate the scope of the problem and to point out alternative mechanisms.

\subsection{Flare of 21 May, 1980}

This major flare began at 20:50 UT and was well-observed by the SMM spacecraft (de Jager and Švestka, 1985). Hoyng et al. (1981) attribute the destabilization of a long filament over an extended polarity-inversion line to the emergence nearby of a bipolar region containing a new pore. Subsequent analysis of magnetograms by Harvey (1982) suggests that the pore formed not by emergence but by the compression of existing flux at the surface. New flux did appear nearby as patches of polarity opposite to their unipolar surroundings. But the net flux directly beneath the activated filament actually decreased. The effect can be seen in the vicinity of the arrow on the magnetogram of $20: 15$ in Figure 4. The sharp alteration of the polarity-inversion line at this location is reflected in the broadening of the neutral-line filament and the appearance of additional fine strands within it; compare the filtergrams at 15:56 and 19:37 UT in Figure 4. The parting of the dark filament is as likely due to the substantial reduction of negativepolarity (black) flux as to the injection of new flux; compare the widening gap in the magnetograms of 20:15 and 21:47 UT in Figure 4 at the location of the arrow. The cancellation of magnetic flux erodes the continuity of the filamentary structure. The filament did not erupt or disappear; instead, $\mathrm{H} \alpha$-emitting material was ejected from one end of the filament in the form of a spray (McCabe et al., 1986).

This flare is a paradigm for the empirical rule first expressed by Martres et al. (1968): evolving magnetic features of one polarity involved in a flare are increasing at the same time as features of the opposite polarity are decreasing. 


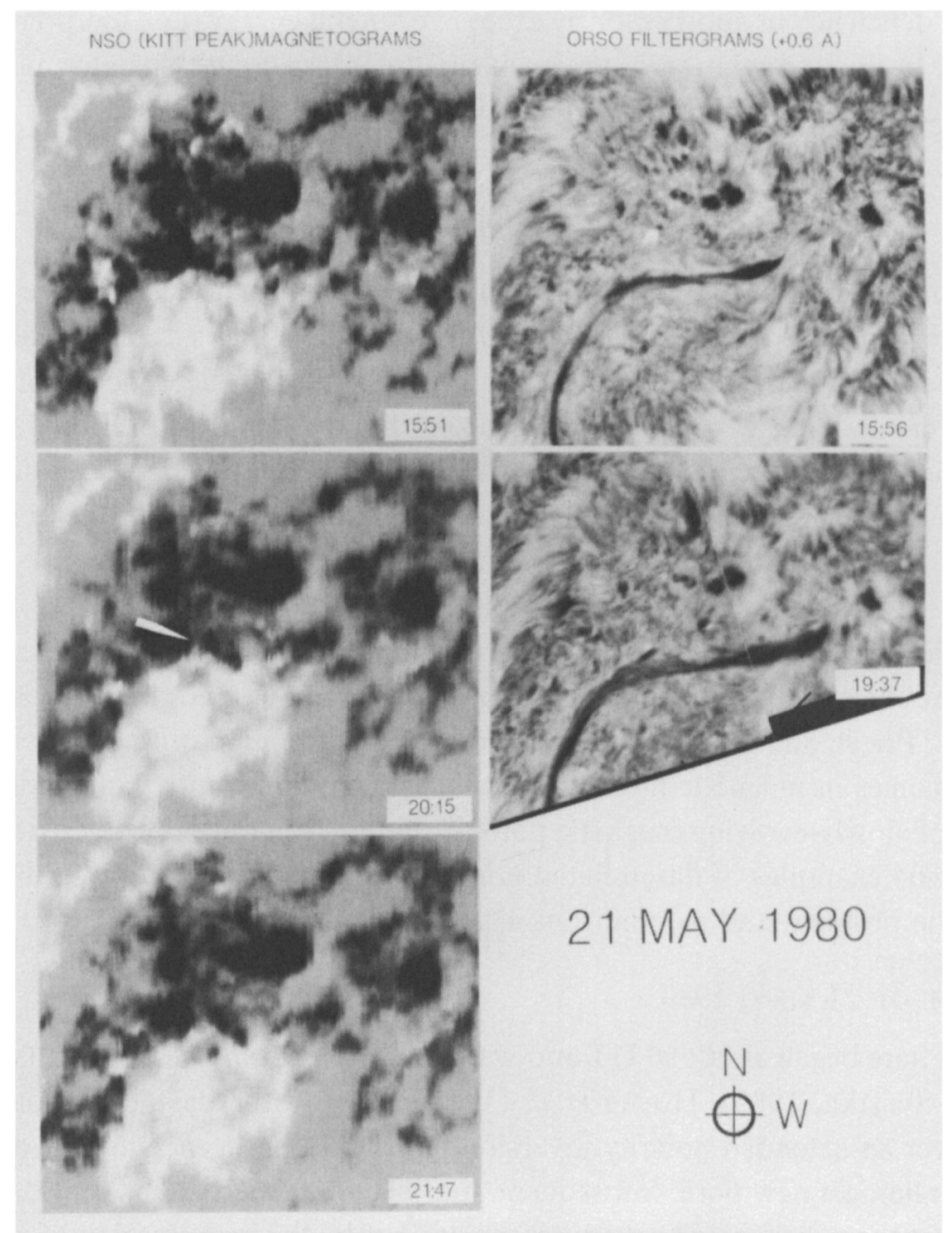

Fig. 4. Changes associated with the major flare beginning at 20:50 UT on 21 May, 1980. Left column: longitudinal component of the magnetic field. Right column: chromospheric fine structure. The arrow on the magnetogram at 20:15 UT points to a widening gap in negative (black) polarity flux. Field of view $=200^{\prime \prime} \times 165^{\prime \prime}$. NSO magnetograms supplied by courtesy of J. W. Harvey.

\subsection{FlaRe of 22 JUNE, 1980}

The activation of a filament in Boulder Region 2517 (Hale 16918) prior to the eruption of a flare (Imp. 2) at 13:05 UT on 22 June, 1980 has been attributed by Simon et al. (1984) to magnetic reconnection between the filament and new emerging flux. The essential circumstances are depicted schematically in Figure 5(b) where pores $O_{8}$ and $O_{9}$ are inferred by Simon et al. to be a new EFR straddling the filament at the polarity inversion line of a large complex of activity. The observed convergence of these pores is, however, contrary to the spreading motion normally associated with an EFR. The chromospheric velocity feature associated with these pores by Raadu et al. (1988) is much weaker than the usual loop flows in an EFR. The designation of $O_{8}-O_{9}$ as an 


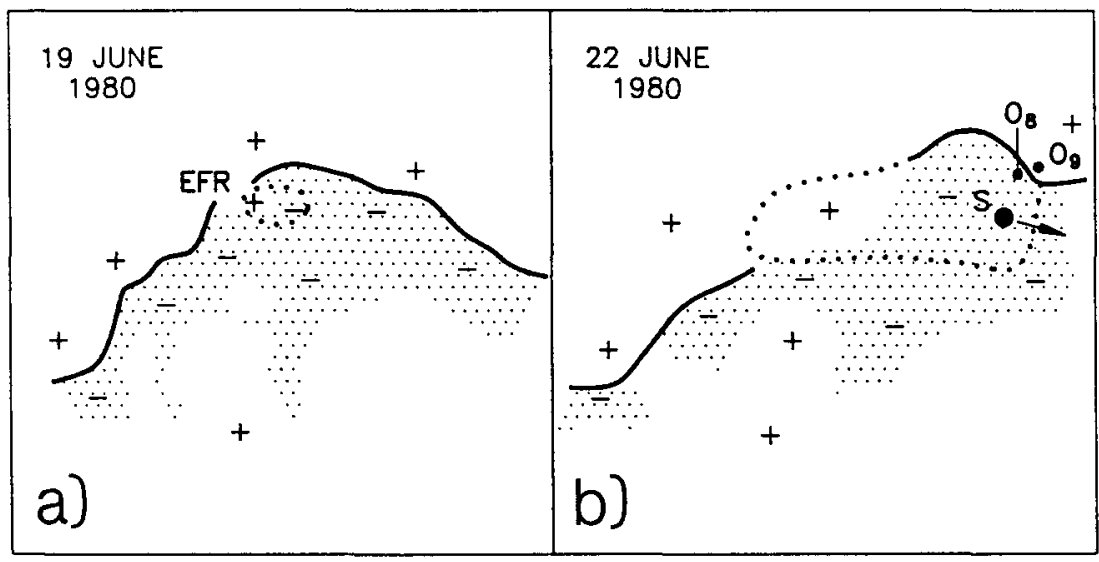

Fig. 5. Schematic view of new bipolar magnetic flux expanding inside an existing complex fo activity. (a) A new EFR (oval outline of heavy dots) appears next to a 'neutral line' filament (full line). (b) After 4 days the new region (outline of heavy dots) has expanded and reshaped the polarity inversion line. Fresh magnetic flux surrounding the westward-moving leader spot $S$ has pushed older flux against the reshaped 'neutral line' near pores $O_{8}$ and $O_{9}$. The stippled area roughly corresponds to the outline of negative magnetic flux in Hale 16918 (Martin et al., 1982). The locations of the filaments on the indicated dates are adapted from Martin et al. (1984).

EFR, or any magnetic connection between these pores is, therefore, debatable. The most significant preflare phenomenon reported by Simon et al. is the very rapid motion of bright $\mathrm{H} \alpha$ emission surrounding $O_{8}$ towards the filament just minutes before the filament erupts and the first flare kernels appear. The moving bright feature may be an early signature of magnetic reconnection but, since the orientation of interacting magnetic fields remains problematical, the circumstances do not lead themselves to a definitive test of the Emerging Flux Model. The small-scale changes noted above are embedded in a much larger evolutionary change (Martin et al., 1984). An EFR forms on 19 June adjacent to the polarity inversion line (Figure 5(a)). As the original EFR expands, another EFR emerges within it. In four days the expanded bipolar region compresses pre-existing magnetic flux at the polarity inversion line and distorts the shape of the original trajectory of the filament (Figure 5(b)). The relative effect on the filament's stability of the small-scale stresses imposed by motion of $O_{8}$ vs large-scale evolutionary stresses driven by emerging flux has yet to be assessed.

\subsection{Flare of 25 June, 1980}

This two-ribbon flare (Imp. 1B) with impulsive phase at 15:51 UT was jointly observed by the VLA, the SMM spacecraft, and ground-based observatories. It was inferred from the detection at $6 \mathrm{~cm}$ wavelength of changing polarized structures $15 \mathrm{~min}$ before the flare that emerging flux might be acting as a trigger (Kundu et al., 1982). Subsequent analysis of photospheric and chromospheric data ruled out emerging flux beneath the location of the polarized microwave-emitting features. It was shown instead that a filament at this location began, more than two hours before the flare, to rise with uniform acceleration and then, at $20 \mathrm{~min}$ before the flare, to execute a more complex series of motions 
including untwisting (Kundu et al., 1985; Gaizauskas, 1984). The preflare untwisting of the filament overlapped in space and time with the changing polarized microwave structures and may be physically related to them.

The prolonged rise of the filament followed by its untwisting and disruption form a single progression with the eventual onset of the flare. Did a particular disturbance initiate the irreversible outward motion of the filament beginning of 13:40 UT? In Figure 6 we examine the chromospheric environment of the filament (enclosed between two arrows) near the beginning of its ascent. The field of view includes only the

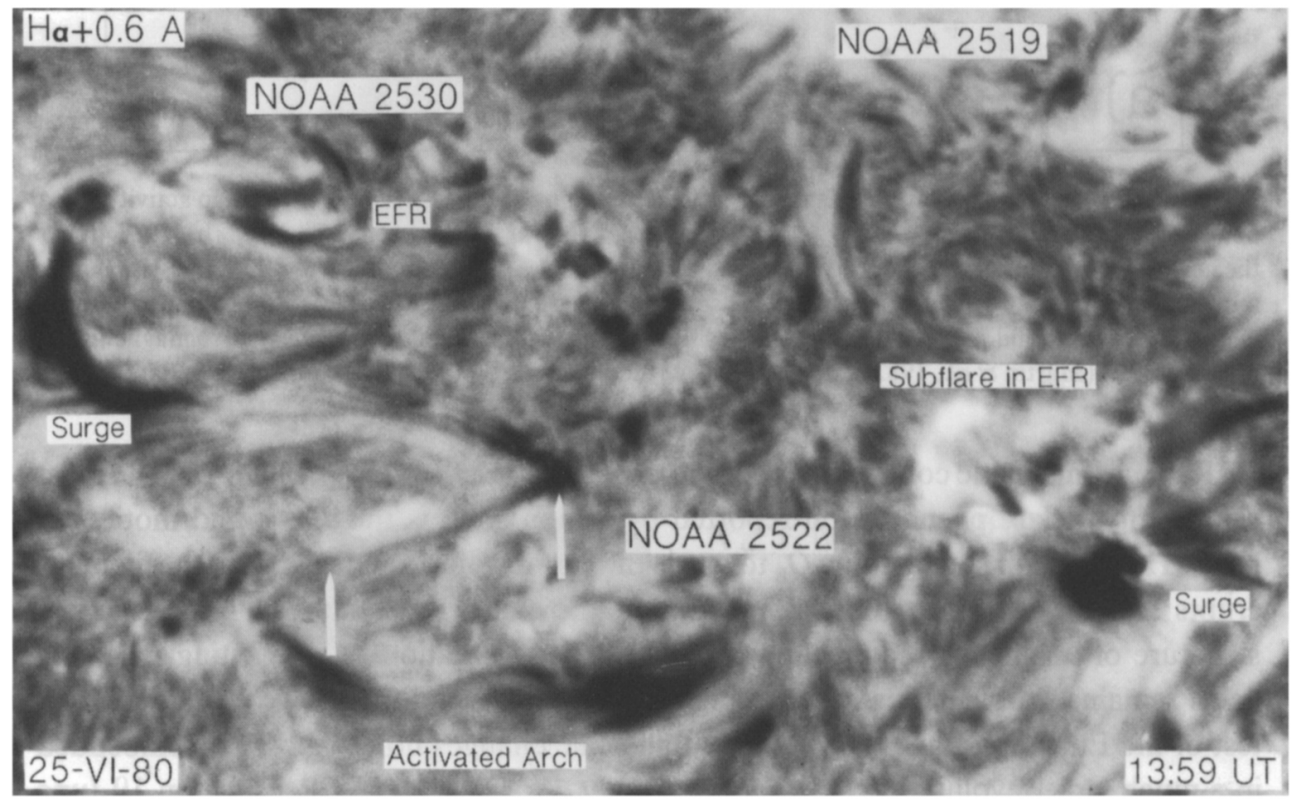

Fig. 6. A close-up view of the chromosphere in part of an activity complex two hours before the small filament enclosed by the arrows erupts immediately prior to a class 1B flare. Dynamic features are labelled and described in the text. Top: north; right: west. Off-band $\mathrm{H} \alpha$ filtergram from the Ottawa River Solar Observatory. Field of view $=290^{\prime \prime} \times 175^{\prime \prime}$.

southeastern corner of the elongated complex of activity which straddles the central meridian in the magnetogram of 22 June, 1980 (Figure 2). The portion of the complex depicted in Figure 6 consists of three numbered bipolar regions in different stages of growth. At the time shown, region 2519 is decaying while 2522 and 2530 each contain vigorously growing EFR. We note the following:

- A flare is erupting inside the EFR of region 2522.

- Surges are in progress in the leading spot of 2522 and the trailing spot of 25530. Surges recur at these locations throughout the day.

- Just below the label 'NOAA 2522' there is a large intrusion in a unipolar area of flux of opposite magnetic polarity (Schmahl, 1982). This isolated pole connects from time to time via an 'activated arch' to the spot which anchors the eastern end of the marked filament. The arch is called 'activated' here because its visibility is strongly 
dependent on wavelenth shifts of the $\mathrm{H} \alpha$ line, implying velocity flows in the structure. The activation in progress in Figure 6 begins at 13:40 UT coincident with the gradual rise in the marked filament but subsides within a half-hour.

- The large spots near either end of the marked filament are moving in opposite directions with the high relative velocity of $0.2 \mathrm{~km} \mathrm{~s}^{-1}$ (Schmahl, 1982).

Any one of the above coincidental phenomena cannot be singled out as 'causing' the initial ascent of the filament. Together, however, they imply a global dynamism in the complex of activity which steadily alters the equilibrium of the filament. The filament tries to readjust by gradually rising until it reaches a state where further equilibrium becomes impossible and a catastrophe results. The 25 June, 1980 filament eruption is an ideal candidate for the application of nonlinear dynamics and the study of chaotic behaviour (Martens, 1984).

On the face of it, emerging flux can be suspected of playing a role during each of the three flare-associated filament eruptions discussed above. But when we probe more deeply, quantitative data which would permit a definitive test of the Emerging Flux Model may elude us, or the flux may emerge remotely to influence the equilibrium of the filament indirectly rather than directly through a current sheet.

\section{Concluding Remarks}

The most challenging aspects of the flare problem deal with the sudden release of energy in a compact volume. A tendency has developed to seek the answers from conditions existing at the affected volume or in a single loop which contains that volume. But observational evidence reviewed here shows that multiple structures are involved in the energy release and that they cannot be considered as uncoupled from a global background. Now, theoretical tools are being developed to cope with a much more complicated and dynamic, hence realistic, magnetic environment than an arcade of loops.

The absence of a singular class of precursor that could reliably foretell a flare, even though observers have sought them assiduously for years, and the disparity among flares (excluding homologues) observed in different active regions, means that preflare transient behaviour does not always arise from the same mechanism. If, as now seems likely, multiple structures are involved in a flare, preflare transient emission may represent a momentarily successful bid by one of several structures to counter a loss of equilibrium. The exact nature of the process depends upon the coupling between structures adjacent to the flare and the rest of the activity complex. This widespread dependence increases the scope for variety among preflare phenomena.

The complexity of the preflare state will not be resolved without many more comprehensive, multi-wavelength analyses of preflare periods observed jointly by spacecraft and ground-based instruments. In those analyses, closer attention should be paid to the way developing large-scale patterns of magnetic fields affect current structures on a local scale. 


\section{References}

Athay, R. G. and Moreton, G. E.: 1961, Astrophys. J. 133, 935.

Athay, R. G., Jones, H. P., and Zirin, H.: 1986, Astrophys. J. 303, 877.

Bai, T.: 1988, Astrophys. J. 328, 860.

Baum, P. J. and Bratenahl, A.: 1980, Solar Phys. 67, 245.

Benz, A. O., Barrow, C. H., Dennis, B. R., Pick, M., Raoult, A., and Simnett, G.: 1983, Solar Phys. 83, 267.

Bruzek, A.: 1967, Solar Phys. 2, 451.

Castenmiller, M. J. M., Zwaan, C., and van der Zalm, E. B. J.: 1986, Solar Phys. 105, 237.

Cheng, C.-C. and Tandberg-Hanssen, E.: 1986, Astrophys. J. 309, 421.

Cheng, C.-C., Tandberg-Hanssen, E., and Orwig, L. E.: 1984, Astrophys. J. 278, 853.

Cheng, C.-C., Tandberg-Hanssen, E., Bruner, E. C., Orwig, L., Frost, K. J., Kenny, P. J., Woodgate, B. E., and Shine, R. A.: 1981, Astrophys. J. 248, L39.

Cheng, C.-C., Bruner, E. C., Tandberg-Hanssen, E., Woodgate, B. E., Shine, R. A., Kenny, P. J., Henze, W., and Poletto, G.: 1982, Astrophys. J. 253, 353.

Cheng, C.-C., Pallavicini, R., Acton, L. W., and Tandberg-Hanssen, E.: 1985, Astrophys. J. 298, 887.

De Jager, C. and Švestka, Z.: 1985, Solar Phys. 100, 435.

De Jager, C., Machado, M. E., Schadee, A., Strong, K. T., Švestka, Z., Woodgate, B.E., and van Tend, W.: 1983, Solar Phys. 84, 204.

Dezsö, L., Gesztelyi, L., Kondas, L., Kovacs, A., and Rostas, S.: 1980, Solar Phys. 67, 317.

Ding, Y. J., Hagyard, M. J., Deloach, A. E., Hong, Q. R., and Liu, X. P.: 1987, Solar Phys. 109, 307.

Dodson, H. W. and Hedeman, E. R.: 1976, Solar Phys. 47, 267.

Fritzová-Švestková, L., Chase, R. C., and Švestka, Z.: 1976, Solar Phys. 48, 275.

Gaizauskas, V.: 1982, Adv. Space Res. 2, No. 11, 11.

Gaizauskas, V.: 1985, in C. de Jager and B. Chen (eds.), Proc. Kunming Workshop on Solar Physics and Interplanetary Travelling Phenomena, Science Press, Beijing, 710.

Gaizauskas, V. and Harvey, K. L.: 1986a, Adv. Space Res. 6, No. 6, 17.

Gaizauskas, V. and McIntosh, P. S.: 1986b, in P. A. Simon, G. Heckman, and M. A. Shea (eds.), SolarTerrestrial Predictions, Proc. Workshop Meudon, France, NOAA/AFGL, p. 126.

Gaizauskas, V., Harvey, K. L., Harvey, J. W., and Zwaan, C.: 1983, Astrophys. J. 265, 1056.

Gesztelyi, L. and Kalman, B.: 1986, Adv. Space Res. 6, No. 6, 29.

Giovanelli, R. G.: 1939, Astrophys. J. 89, 555.

Hagyard, M. J.: 1988, Solar Phys. 115, 107.

Hagyard, M. J. and Rabin, D. M.: 1986, Adv. Space Res. 6, No. 6, 7.

Hagyard, M. J., Smith, J. R., Jr., Teuber, D., and West, E. A.: 1984, Solar Phys. 91, 115.

Harrison, R. A.: 1986, Astron. Astrophys. 162, 283.

Harrison, R. A., Waggett, P. W., Bentley, R. D., Phillips, K. J. H., Bruner, M., Dryer, M., and Simnett, G. M.: 1985, Solar Phys. 97, 387.

Harvey, J. W.: 1982, Adv. Space Res. 2, No. 11, 31.

Hénoux, J.-C. and Somov, B. V.: 1987, Astron. Astrophys. 185, 306.

Heyvaerts, J., Priest, E. R., and Rust, D. M.: 1977, Astrophys. J. 216, 123.

Hoyng, P. and 11 co-authors: 1981, Astrophys. J. 246, L155.

Hurford, G. J. and Zirin, H.: 1982, AFGL-TR-82-0117.

Hurford, G. J., Read, J. B., and Zirin, H.: 1984, Solar Phys. 94, 413.

Kahler, S.: 1979, Solar Phys. 62, 347.

Kai, K., Nakajima, H., and Kosugi, T.: 1983, Publ. Astron. Soc. Japan 35, 285.

Kovacs, A. and Dezsö, L.: 1986, Adv. Space Res. 6, No. 6, 29.

Krall, K. R., Smith, J. B., Jr., Hagyard, M. J., West, E. A., and Cummings, N. T.: 1982, Solar Phys. 79, 59.

Kundu, M. R.: 1965, Solar Radio Astronomy, John Wiley Interscience, New York.

Kundu, M. R.: 1986, Adv. Space Res. 6, No. 6, 93.

Kundu, M. R. and Shevgaonkar, R. K.: 1985, Astrophys. J. 291, 860.

Kundu, M. R., Rust, D. M., and Bobrowsky, M.: 1983, Astrophys. J. 265, 1084.

Kundu, M. R., Schmahl, E. J., and Velusamy, T.: 1982, Astrophys. J. 253, 963.

Kundu, M. R., Schmahl, E. J., Velusamy, T., and Vlahos, L.: 1982, Astron. Astrophys. 108, 188.

Kundu, M. R., Gaizauskas, V., Woodgate, B. E., Schmahl, E. J., Shine, R., and Jones, H. P.: 1985, Astrophys. J. Suppl. 57, 621. 
Kundu, M. R., Gopalswamy, N., Saba, J. L. R., Schmelz, J. T., and Strong, K. T.: 1987, Solar Phys. 114, 273. Lang, K. R.: 1980, in R. F. Donnelly (ed.), Solar-Terrestrial Predictions Proceedings III, Solar Activity Predictions, US Dept. of Commerce, p. C-131.

Lang, K. R. and Willson, R. F.: 1986, Adv. Space Res. 6, No. 6, 97.

Lin, Y.Z. and Gaizauskas, V.: 1987, Solar Phys. 109, 81.

Low, B. C.: 1982, Rev. Geophys. Space Phys. 20, 145.

Low, B. C. and Wolfson, R.: 1988, Astrophys. J. 324, 547.

Machado, M. E.: 1985, Solar Phys. 99, 159.

Machado, M. E., Orwig, L. E., and Antonucci, E.: 1986, Adv. Space Res. 6, No. 6, 101.

Machado, M. E., Somov, B. V., Rovira, M. G., and de Jager, C.: 1983, Solar Phys. 85, 157.

Machado, M. E., Moore, R. L., Hernandez, A. M., Rovira, M. G., Hagyard, M. J., and Smith, J. B., Jr.: 1988, Astrophys. J. 326, 425.

Marsh, K. A.: 1978, Solar Phys. 59, 105.

Martens, P. C. H.: 1984, Phys. Reports 115, 315.

Martin, S. F.: 1980, Solar Phys. 68, 217.

Martin, S. F., Livi, S. H. B., and Wang, J.: 1985, Australian J. Phys. 38, 929.

Martin, S. F., Dezsö, L., Antalová, A., Kučera, A., and Harvey, K. L.: 1982, Adv. Space Res. 2, No. 11, 39.

Martin, S. F. and 11 co-authors: 1984, Adv. Space Res. 4, No. 7, 61.

Martres, M.-J., Michard, R., Soru-Iscovici, I., and Tsap, T.: 1968, in K. O. Kiepenheuer (ed.), 'Structure and Development of Solar Active Regions', IAU Symp. 35, 311.

Martres, M.-J., Rayrole, J., Semel, M., Soru-Escaut, I., Tanaka, K., Makita, M., and Moriyama, F.: 1982, Publ. Astron. Soc. Japan 34, 299.

Martres, M.-J., Woodgate, B. E., Mein, N., Mouradian, Z., Rayrole, J., Schmieder, B., Simon, G., and Soru-Escaut, I.: 1984, Adv. Space Res. 4, No. 7, 5.

McCabe, M. K., Švestka, Z., Howard, R. A., Jackson, B. V., and Sheeley, N. R.: 1986, Solar Phys. 103, 399.

McIntosh, P. S. and Wilson, P. R.: 1985, Solar Phys. 97, 59.

Moreton, G. E.: 1961, Sky Telesc. 21, 145.

Mouradian, Z., Martres, M.-J., and Soru-Escaut, I.: 1983, Solar Phys. 87, 309.

Mouradian, Z., Martres, M.-J., Soru-Escaut, I., and Gesztelyi, L.: 1987, Astron. Astrophys. 183, 129.

Nagy, I.: 1983, Publ. Debrecen Obs. 5, 107.

Nakajima, H., Dennis, B. R., Hoyng, P., Nelson, G., Kosugi, T., and Kai, K.: 1985, Astrophys. J. 288, 806.

Ogir, M. B.: 1981, Izv. Krymsk. Astrofiz. Obs. 64, 118.

Porter, J. G., Toomre, J., and Gebbie, K. B.: 1984, Astrophys. J. 283, 879.

Porter, J. G., Moore, R. L., Reichmann, E. J., Engvold, O., and Harvey, K. L.: 1987, Astrophys. J. 323, 380.

Priest, E. R. (ed.): 1981, Solar Flare Magnetohydrodynamics, Gordon and Breach, London.

Priest, E. R.: 1982, Solar Magnetohydrodynamics, D. Reidel Publ. Co., Dordrecht, Holland.

Priest, E. R.: 1985, Rep. Prog. Phys. 48, 955.

Priest, E. R., Gaizauskas, V., Hagyard, M. J., Schmahl, E. J., and Webb, D. F.: 1986, in M. R. Kundu and

B. E. Woodgate (eds.), Energetic Phenomena on the Sun, NASA CP-2439, Ch. 1.

Raadu, M. A., Schmieder, B., Mein, N., and Gesztelyi, L.: 1988, Astron. Astrophys. 197, 289.

Rabin, D. M., Moore, R. L., and Hagyard, M. J.: 1984, Astrophys. J. 287, 404.

Raoult, A., Pick, M., Dennis, B. R., and Kane, S. R.: 1985, Astrophys. J. 299, 1027.

Rust, D. M. and Roy, J.-R.: 1975, AFCRL-TR-0437, 61.

Rust, D. M., Nakagawa, Y., and Neupert, W. M.: 1975, Solar Phys. 41, 392.

Sattarov, I.: 1983, Astron. Zh. 60, 350.

Schadee, A., de Jager, C., and Švestka, Z.: 1983, Solar Phys. 89, 287.

Schmahl, E. J.: 1982, Adv. Space Res. 2, No. 11, 73.

Simnett, G. M. and Harrison, R. A.: 1985, Solar Phys. 99, 291.

Simon, G., Mein, N., Mein, P., and Gesztelyi, L.: 1984, Solar Phys. 93, 325.

Soru-Escaut, I., Martres, M.-J., and Mouradian, Z.: 1985, Astron. Astrophys. 145, 19.

Spicer, D. S.: 1976, An Unstable Arch Model of a Flare, NRL report 8036.

Spicer, D. S.: 1982, Space Sci. Rev. 31, 351.

Spicer, D. S. and Brown, J. C.: 1981, in S. Jordan (ed.), The Sun as a Star, NASA SP-450.

Švestka, Z.: 1976, Solar Flares, D. Reidel Publ. Co., Dordrecht, Holland.

Tang, F.: 1983, Solar Phys. 89, 43.

Thomas, R. J. and Teske, R. G.: 1971, Solar Phys. 16, 431. 
Van Hoven, G.: 1976, Solar Phys. 49, 95.

Van Hoven, G. and Hurford, G. J.: 1986, Adv. Space Res. 6, No. 6, 83.

Van Hoven, G. and 18 co-authors: 1980, in P. Sturrock (ed.), Solar Flares, Colorado Associated University Press, Boulder, Ch. 1.

Vlahos, L., Machado, M. E., Ramaty, R., and Murphy, R. J.: 1986, in M. R. Kundu and B. E. Woodgate (eds.), Energetic Phenomena on the Sun, NASA CP-2439, Ch. 2.

Webb, D. F.: 1985, Solar Phys. 97, 321.

Willson, R. F.: 1983, Solar Phys. 83, 285.

Willson, R. F.: 1984, Solar Phys. 92, 189.

Willson, R. F. and Lang, K.R.: 1984, Astrophys. J. 279, 427.

Wolfson, C. J.: 1982, Solar Phys. 76, 377.

Woodgate, B. E.: 1982, Adv. Space Res. 2, No. 11, 61.

Woodgate, B. E. and 15 co-authors: 1981, Astrophys. J. 244, L133.

Woodgate, B. E. and 8 co-authors: 1984, Adv. Space Res. 4, No. 7, 5.

Zirin, H.: 1983, Astrophys. J. 274, 900.

Zirin, H. and Liggett, M. A.: 1987, Solar Phys. 113, 267.

Zirin, H. and Tanaka, K.: 1973, Solar Phys. 32, 173.

Zwaan, C.: 1987, Ann. Rev. Astron. Astrophys. 25, 83. 